SESSION 3

OBSERVATIONS OF OTHER PULSARS 


\title{
3.1 The galactic POPULATION OF PULSARS
}

\author{
M. I. LAR GE*
}

University of Manchester, Nuffield Radio Astronomy Laboratory, Jodrell Bank, U.K.

\begin{abstract}
This paper examines the distribution of pulsars in the Galaxy and compares it with the distribution of supernova remnants. The dispersion measure has been used as a distance indicator to obtain the distribution in luminosity, $z$-distance and period of the pulsars. The $z$-distance distribution is similar within the uncertainties to that of the supernova remnants. An estimate of the total number of pulsars in the Galaxy with a peak luminosity $\geqslant 1 \mathrm{fu}(\mathrm{dm})^{2}$ gives $5 \times 10^{5}$ within a factor of 10 . The data is consistent with the hypothesis that pulsars are formed at the same rate as supernovae, if the pulsars are assumed to have a timescale of $10^{7} \mathrm{yr}$.
\end{abstract}

\section{Introduction}

The extent to which pulsars and supernovae are physically associated is uncertain. It is now generally accepted that pulsars are beamed radiation from neutron stars, and a supernova explosion has been thought to provide suitable conditions for the formation of neutron stars (Colgate and White, 1966) although there are theoretical difficulties (Arnett, 1969). It is therefore of considerable interest to determine whether pulsars and supernovae do have a common origin. The direct observational evidence is unsatisfactory: of 55 pulsars** and about 90 known or suspected supernova remnants (see for example, Milne, 1970) there is one unequivocal association (NP 0532 with the Crab Nebula) and one probable but by no means certain association (PSR 0833-45 with the Vela remnant).

Several factors could contribute to the lack of obvious correlation between pulsars and supernovae. The detectable traces of a supernova explosion seem to last for about $10^{5} \mathrm{yr}$, whereas most pulsar ages derived from the rate of change of period are at least an order of magnitude greater than this (Ostriker and Gunn, 1969). It is quite possible that the comparatively young pulsars in known remnants have not been found simply because adequate sensitivity has not been achieved for short period pulsars (Large, 1970a). Furthermore a large proportion of all pulsars may be missed on account of their orientation if the beam of the pulsar is restricted in the plane containing the rotation axis. It is also possible that pulsars are formed with high velocity so that they appear coincident with the parent supernova for a short fraction of their lifetime only. Using this idea Prentice (1970) has suggested that several pulsars might be associated with near (but not coincident) supernovae. It is interesting that the two longest period pulsars (NP 0527, JP 2319) are fairly near the two most spectacular supernovae, although physical association in both cases seems improbable.

From the foregoing remarks, it appears that despite the paucity of individual associations, supernovae could well be the origin of some or all pulsars. A phe-

* On leave of absence from Department of Physics, University of Sydney, Australia.

** A recent compilation by Maran and Modali (1970) includes 50 pulsars. A further 5 were reported by Davies et al. (1970). 
nomenological analysis of radio evolution of pulsars by Aizu and Taketani (1970) supports this hypothesis. The main objective of this talk is to examine the distribution and number of pulsars in the Galaxy to find out whether they are consistent with the probable rate of production of supernovae in the Galaxy. With the comparatively small sample of pulsars so far discovered (55) it is not possible to deduce the finer details of the galactic distribution. I have therefore chosen three parameters which seem to me of particular importance namely the pulsar period $(P)$, the absolute radio luminosity $(L)$ and the height above the plane of the Galaxy $(z)$ and attempted to derive the spatial density of pulsars $\varrho(P, L, z)$ in terms of these three quantities. The first part of the talk is concerned with the analysis of the available data and discussion of the likely sources of error in the derived distributions. The second half is more speculative in nature, being devoted to astrophysical interpretation of these results and comparison with the supernova data. In a series of papers on the nature of pulsars Gunn and Ostriker (1970) formulated statistical methods for the analysis of pulsar observations based on their magnetic dipole model, and applied this formalism to the extant observations. It is interesting that many of the results that I derive without assuming a theoretical model of pulsars are in broad agreement with Gunn and Ostriker's conclusions.

\section{The Data}

In order to derive the galactic density of pulsars from the observed numbers, it is necessary to have a means of determining pulsar distances, and to know the instrumental selection effects in terms of such quantities as luminosity, period, dispersion measure and galactic $z$-distance.

The distance to each pulsar, in terms of the integrated electron density in the line-ofsight, is known from the dispersion measure, since it is unlikely that an appreciable fraction of the dispersion arises in the source (Rickett, 1969). Many workers have estimated the mean electron density in the Galaxy in order to obtain a calibration of the dispersion measure distance scale. Prentice and ter Haar (1970) in particular have studied the distribution of $\mathrm{H}$ II regions within $1 \mathrm{kpc}$ of the Sun in order to determine their individual contribution to the dispersion of pulsars, and this type of approach is undoubtedly valuable in determining the distance to particular pulsars. However their work shows that a single $\mathrm{H}$ II region rarely contributes as much as $50 \%$ of the total pulsar dispersion, suggesting that the dispersion measure is quite a good statistical measure of pulsar distances. In the work described in this paper I have used the dispersion measure as the distance measure, deferring to the later sections the question of relating these distances to true geometrical distances.

The assessment of search selection effects is more difficult. Most pulsars have been discovered as individual spikes on chart records, with a sensitivity which depends in a complicated way on the response of the pen, the statistics of the pulse amplitudes and the quickness of the observer's eye. Furthermore many pulsars in the northern sky have been found at relatively low radio frequencies $(\sim 100 \mathrm{MHz})$ where dispersion broadening of the pulses in the receiver bandwidth greatly reduces the sensitivity to 
highly dispersed pulsars. It seems impossible to make proper allowance for these complicated selection effects in the various searches, and I have made no attempt to do so. Instead I have used the relatively homogeneous sample of 29 pulsars discovered at the Molonglo Radio Observatory. The Molonglo pulsars were discovered in uniform and well calibrated searches of about 7 ster of the southern sky. The Molonglo Cross operates at $408 \mathrm{MHz}$, and most of the searches were made using the full $4 \mathrm{MHz}$ bandwidth and split beams of the E-W arm. Most of the accessible longitudes of the galactic plane were further searched between $\pm 10^{\circ}$ in latitude using 'dispersion removers' to extend the sensitivity to dispersion measures of about $400 \mathrm{~cm}^{-3} \mathrm{pc}$. The confining of this latter search to the Galaxy does not involve a selection, since pulsars of dispersion measure greater than $50 \mathrm{~cm}^{-3} \mathrm{pc}$ are not found more than $10^{\circ}$ from the galactic plane. The Molonglo searches have been calibrated by generating artificial pulsar signals of known intensity and assessing the limiting mean flux density by examination of chart records using the same procedures used in finding real pulsars. Despite the subjective aspects of this approach, the calibration is self-consistent within about $1 \mathrm{~dB}$ since independent observers were in agreement in determining the limiting flux densities. Data are thus available on the sensitivity of the Molonglo searches in terms of period and dispersion measure. The main results of this calibration have been published in graphical form (Large, 1970b) and further details will be given in due course (Large and Vaughan, 1971).

The observed distributions of the 29 Molonglo pulsars in period, luminosity, and $z$-distance are shown in Figures 1, 2 and 3. As explained in the introduction, dispersion measure has been used as a measure of distance so that $z$-distance is plotted in units

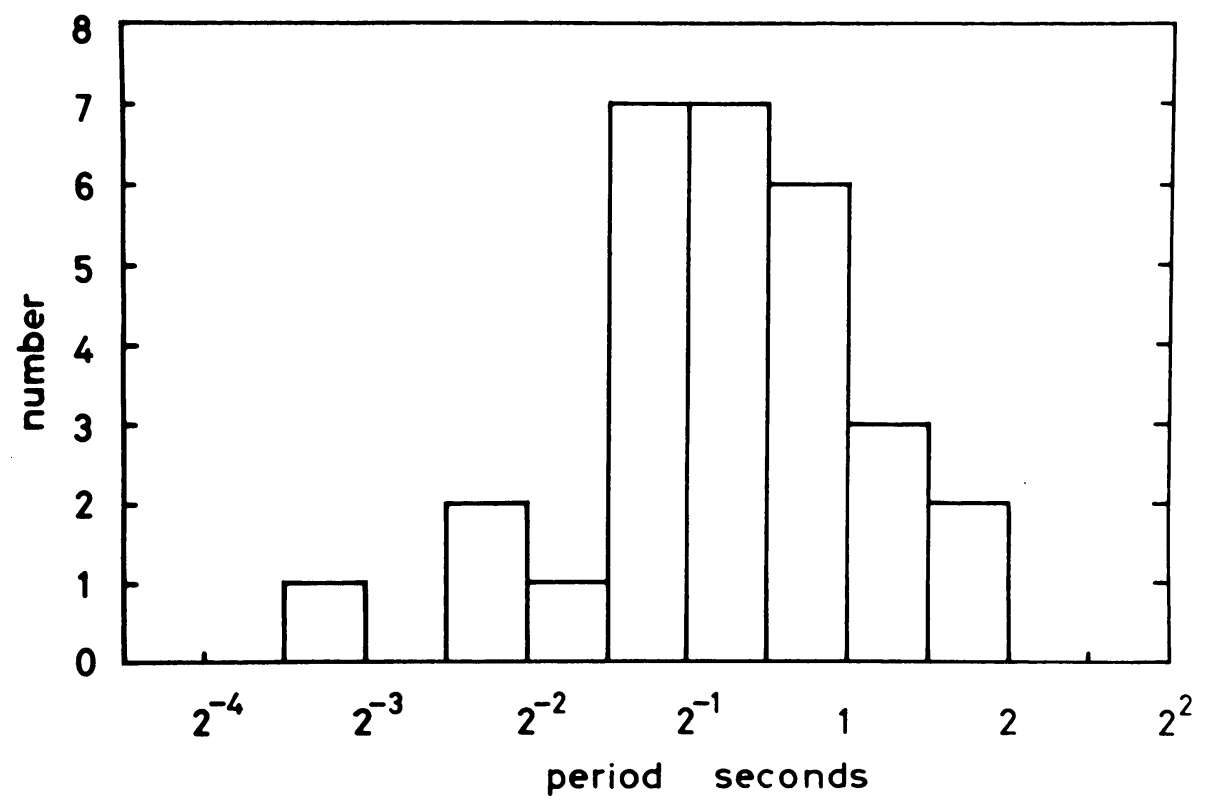

Fig. 1. The observed distribution in period of 29 Molonglo pulsars. 
of $\mathrm{cm}^{-3} \mathrm{pc}$. The luminosity is defined as the flux density at $408 \mathrm{MHz}$ averaged over the entire period multiplied by the square of the dispersion measure. The unit of luminosity is therefore $10^{-26} \mathrm{Wm}^{-2} \mathrm{~Hz}^{-1}\left(\mathrm{~cm}^{-3} \mathrm{pc}\right)^{2}$ which is abbreviated to $\mathrm{fu}(\mathrm{dm})^{2}$ throughout this paper. In drawing the histograms of Figures 1,2 and 3 the intervals of 0.5 in $\log _{2} P, 0.5$ in $\log _{10} L$, and $2 \mathrm{~cm}^{-3}$ pc in $z$ were chosen to divide the pulsars into about 5 groups in each distribution. The distributions represent the true galactic distributions of the pulsars as modified by the selection effects in the searches, with large statistical errors.

Several workers have drawn attention to correlations between pulsar parameters. In particular there is apparently a weak tendency for higher latitude pulsars to have

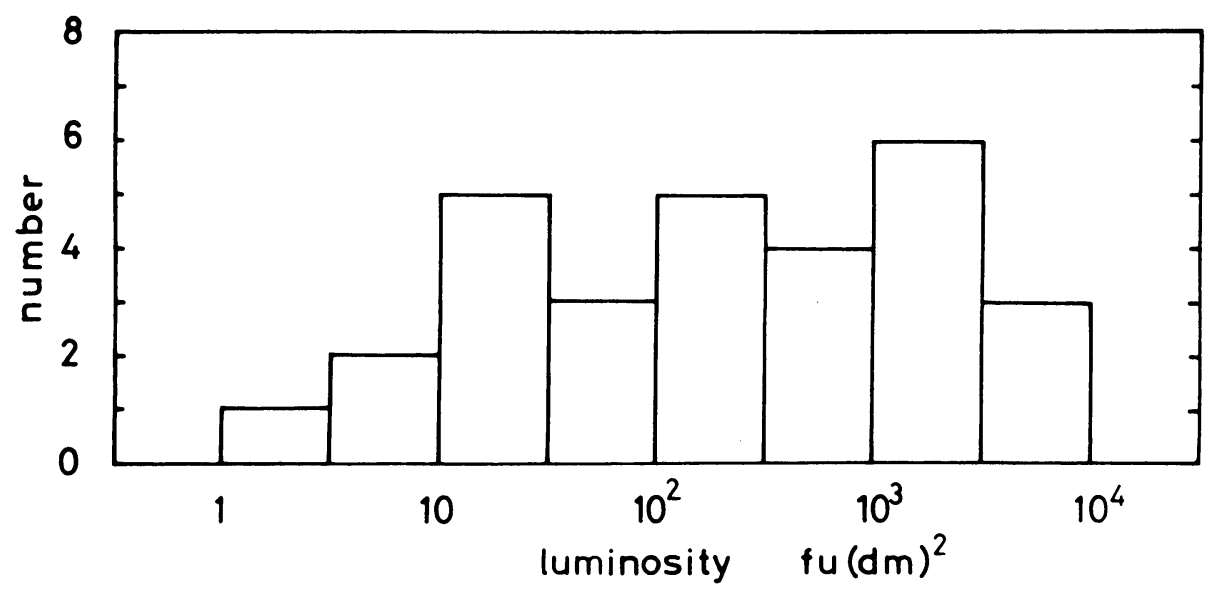

Fig. 2. The observed distribution in radio luminosity of 29 Molonglo pulsars. The luminosity is based on the pulsar mean flux density and is expressed in units of $10^{-26} \mathrm{Wm}^{-2} \mathrm{~Hz}^{-1}\left(\mathrm{~cm}^{-3} \mathrm{pc}\right)^{2}$.

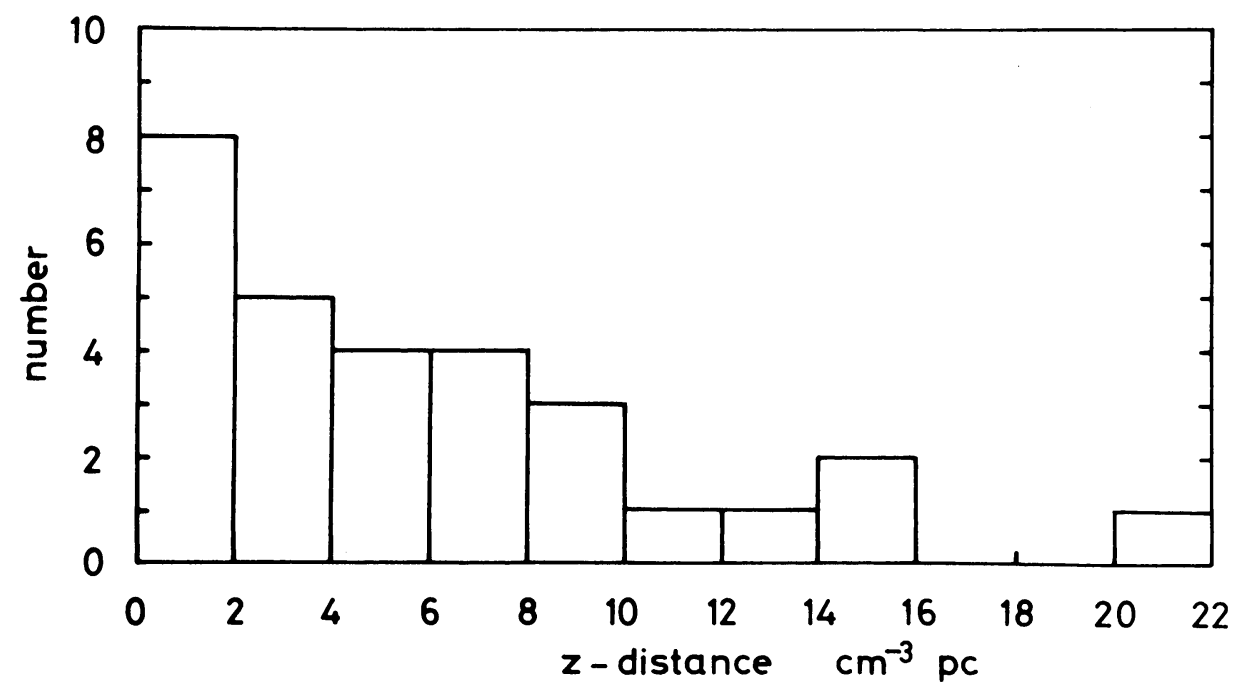

Fig. 3. The observed distribution in galactic $z$-distance of 29 Molonglo pulsars. 
longer periods, and this has been interpreted as indicating that longer period pulsars have lower absolute luminosities (Gold, 1970; Notni et al., 1970). Certainly such correlations are to be expected if pulsars typically become weaker and increase in period as they age. However, the observed correlations are weak, subject to selection effects and barely detectable among the Molonglo pulsars. For example, in Figure 4, the periods and luminosities of the Molonglo pulsars are plotted as a scatter diagram. The correlation coefficient between $\log P$ and $\log L$ is 0.30 which is significant at the

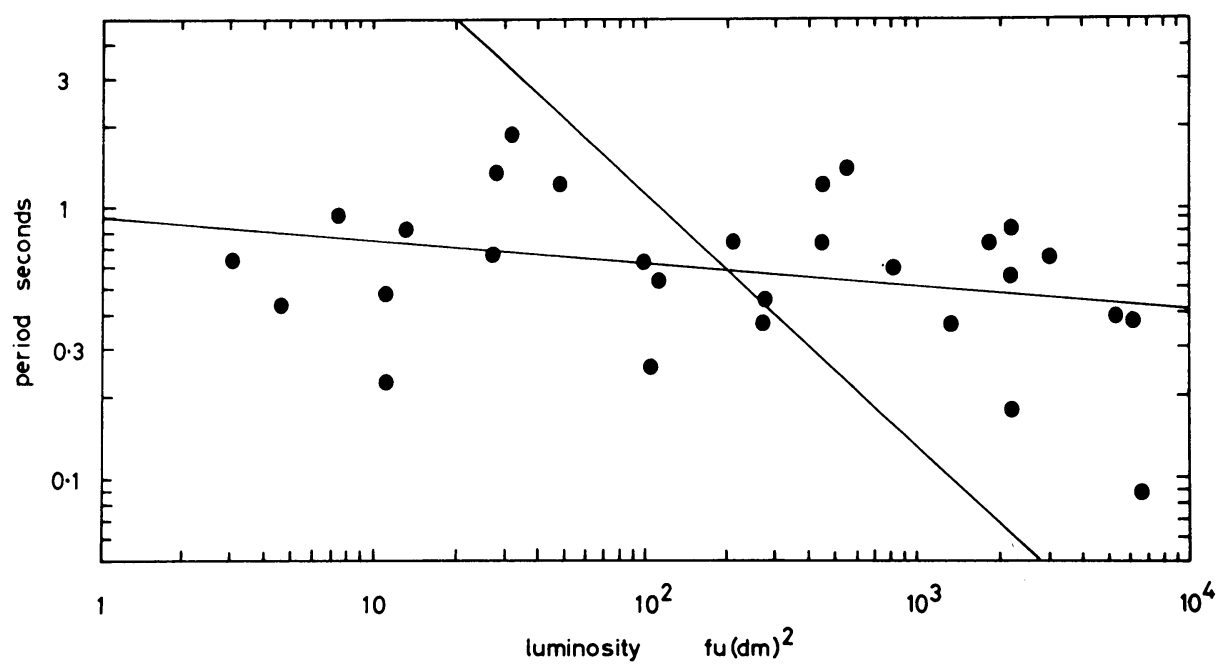

Fig. 4. Diagram showing the period and luminosity of 29 Molonglo pulsars. The correlation between $\log P$ and $\log L$ is barely significant, and is largely produced by one pulsar. The two regression lines are shown.

$10 \%$ level only. The two regression lines of $\log P$ on $\log L$ and vice versa are shown on the diagram. Most of the correlation is produced by one pulsar (PSR 0833-45, period $0.089 \mathrm{sec}$ ). The regression lines show that pulsars of period $\sim 0.3 \mathrm{sec}$ perhaps have luminosities about 3 times that for periods of about $1 \mathrm{sec}$. Similarly, over the full range of luminosity plotted, the periods change by perhaps a factor of 2 . However, over the observed ranges of period and luminosity, the systematic variation of period with luminosity is considerably less than the intrinsic scatter of the observed periods and luminosities.

There is a systematic difference between the Molonglo pulsars and the rest in this respect. Molonglo pulsars are of shorter period and lower latitude than the others. This difference accounts for most of the correlation between latitude and period, and hence needs examining carefully for instrumental selection effects.

\section{Method of Analysis}

The number of pulsars observed in ranges $\mathrm{d} P, \mathrm{~d} L$ and $\mathrm{d} z$ of period, luminosity and 
$z$-distance can be written

$$
N(P, L, z) \mathrm{d} P \mathrm{~d} L \mathrm{~d} z=A(P, L, z) \varrho(P, L, z) \mathrm{d} P \mathrm{~d} L \mathrm{~d} z
$$

where $A(P, L, z) \mathrm{d} z$ is the volume of space effectively explored at period $P$, luminosity $L$ and $z$ distance between $z$ and $z+\mathrm{d} z$. In principle the required spatial density $\varrho(P, L, z)$ can be derived from the observed distributions and the sensitivity factor $A(P, L, z)$. It is clear however that without some simplifying assumptions this cannot be done adequately on the basis of 29 (nor even 55) pulsars.

As a first approximation, the density is written as the product of three independent functions,

$$
\varrho(P, L, z)=\varrho_{0} \varrho_{1}(P) \varrho_{2}(L) \varrho_{3}(z)
$$

Separation of the variables in this way makes it possible to derive estimates of the galactic distribution. It is justified only in so far as the respective distributions are uncorrelated. It will be shown that the small degree of correlation indicated by Figure 4 introduces negligible errors in the derived distributions compared with the statistical errors.

From Equations 1 and 2 the distribution in period can now be written

$$
\varrho_{1}(P) \propto \frac{\iint N(P, L, z) \mathrm{d} L \mathrm{~d} z}{\iint A(P, L, z) \varrho_{2}(L) \varrho_{3}(z) \mathrm{d} L \mathrm{~d} z}
$$

with similar expressions for $\varrho_{2}(L)$ and $\varrho_{3}(z)$. These equations have been solved iteratively using the observed distributions of Molonglo pulsars (Figures 1, 2 and 3) and the data on the search calibrations to compute the sensitivity function $A(P, L, z)$. The resulting derived distributions $\varrho_{1}(P), \varrho_{2}(L)$ and $\varrho_{3}(z)$ are shown in Figures 5, 6 and 7 , with distance measured in dispersion units of $\left(\mathrm{cm}^{-3} \mathrm{pc}\right)$ and luminosity in flux units (dispersion measure) ${ }^{2}$ as explained previously.

\section{Result and Discussion of Errors}

It will be seen from the previous section that each distribution, $\varrho_{1}(P), \varrho_{2}(L)$ and $\varrho_{3}(z)$ is derived from the corresponding observed distribution by dividing by a scaling function. For example Equation 3 can be written

$$
\varrho_{1}(P)=\frac{1}{D(P)} N(P)
$$

where $N(P)$ is the observed distribution in period and $D(P)$ is the scaling function computed from the known sensitivity and the other two distributions $\varrho_{2}(L)$ and $\varrho_{3}(z)$.

The observed distributions $N(P), N(L)$ and $N(z)$ contain large random errors as they are based on only 29 pulsars. The random errors in the derived distributions are largely caused by these random errors in the corresponding observed distribution. 


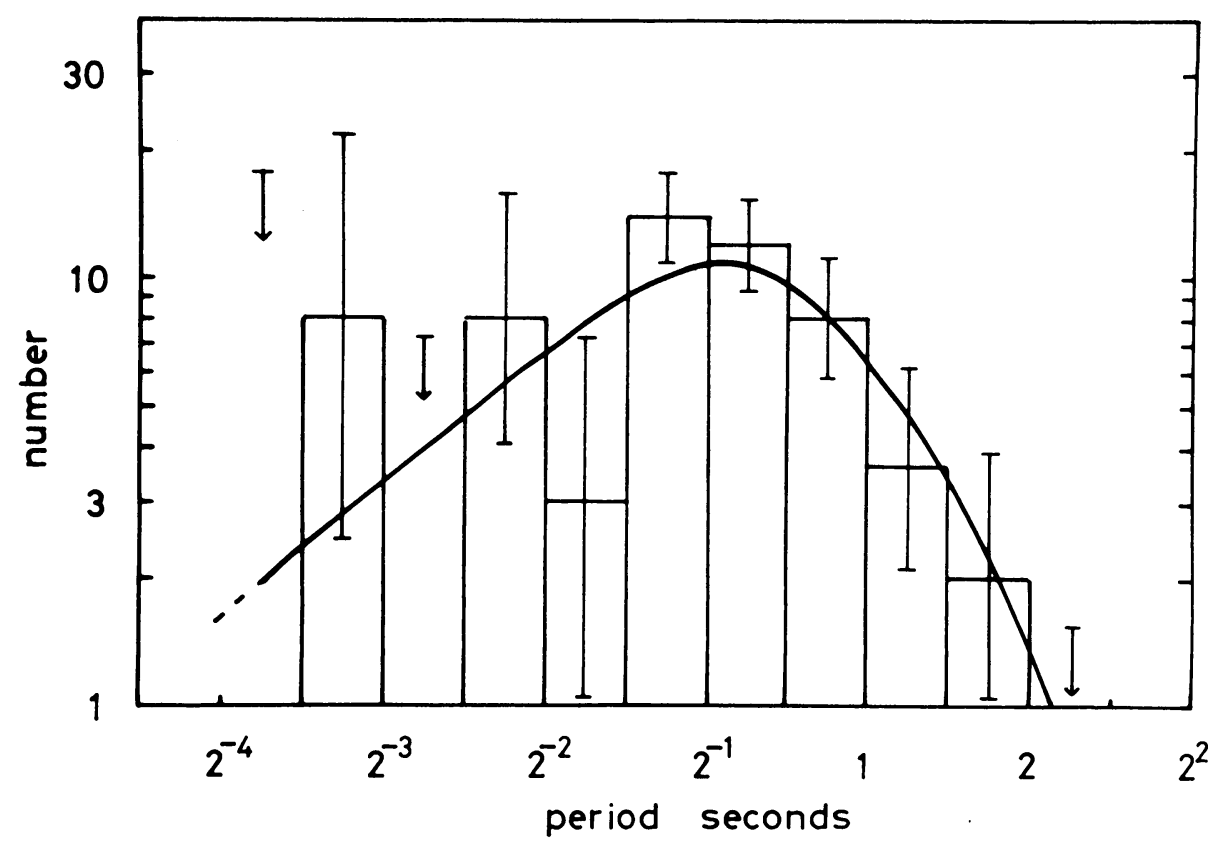

Fig. 5. The distribution, $Q_{1}(P)$, of pulsars in period derived from the observed distribution of the Molonglo pulsars (Figure 1) and a scaling function calculated from the measured sensitivity data and the distributions of pulsars in luminosity and $z$-distance. The probable error limits reflect sampling errors in the observed distribution, and are calculated from cumulative sums of the Poisson distribution.

The random errors in the scaling denominators are relatively small as the denominators are relatively insensitive to changes in the assumed distributions. The random errors indicated in Figures 5 and 6 are 'probable error' points determined by assuming that the number of pulsars observed in each class is Poisson distributed. Systematic errors are discussed for each distribution separately.

\section{A. THE DISTRIBUTION IN PERIOD}

The derived distribution in period differs somewhat from the observed distribution. In particular the relative abundance of short period pulsars is considerably greater than the observed distribution indicates. This conclusion supports a less complete analysis which showed (Large, 1970b) that for periods of $\sim 0.2 \mathrm{sec}$ the Molonglo search might be missing more than half of the pulsars within the theoretical sensitivity limit. At the long period end of the distribution the sharp cut-off appears to be real, as the Molonglo search maintained good sensitivity to well beyond 4 s period.

The assumption that period is independent of luminosity might have introduced systematic distortion into the derived period distribution. If in fact the period changes by a factor of about 2 over the observed range of luminosity as suggested by Figure 4, then the true period distribution would be shifted towards longer periods for low luminosities, and towards shorter periods for high luminosities, with the mean curve 


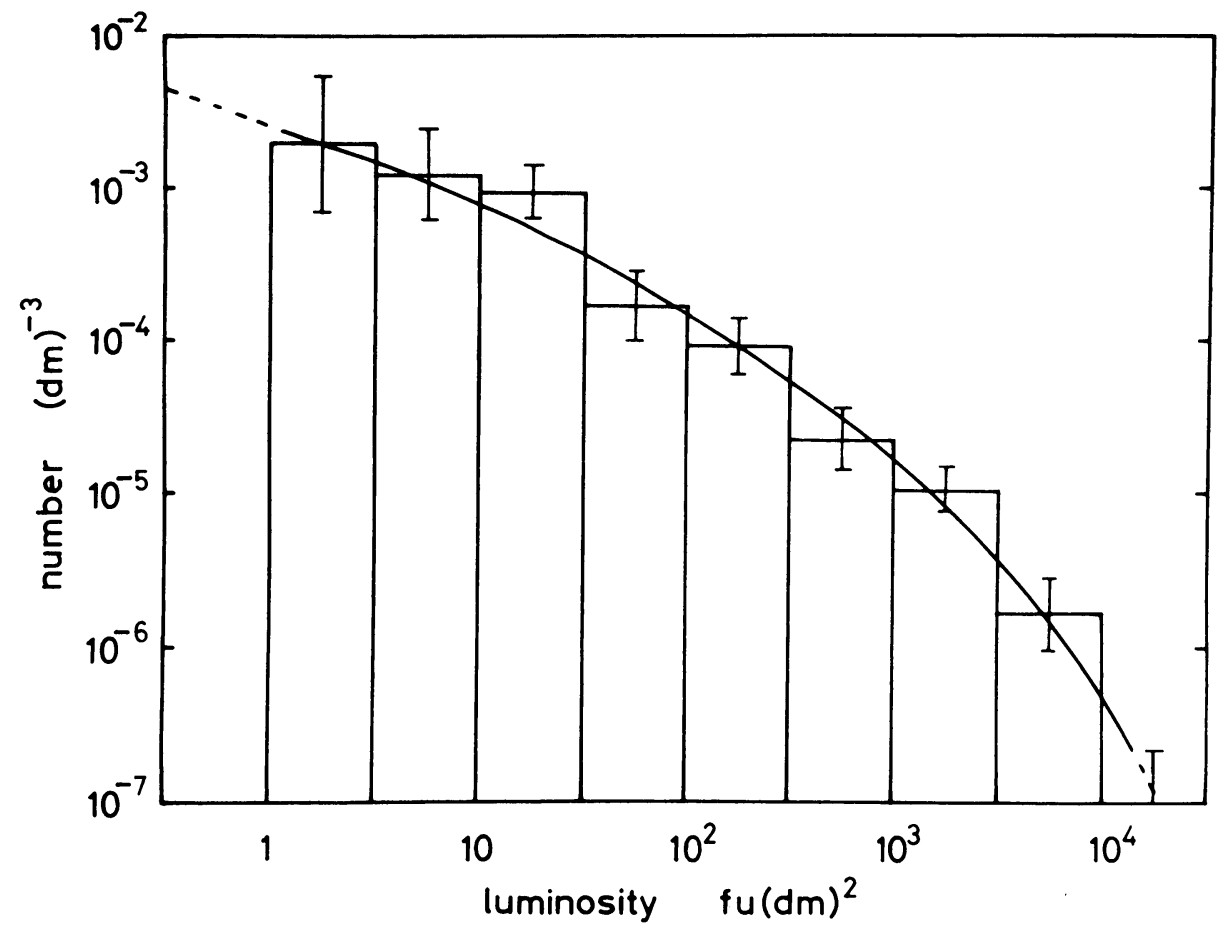

Fig. 6. The distribution $\varrho_{2}(L)$, of pulsars in absolute luminosity derived from the observed distribution of Molonglo pulsars (Figure 2). The histogram shows the number of pulsars per unit volume in each range of luminosity, with the probable sampling errors. As elsewhere, 'distance' is measured in terms of dispersion measure in units of $\mathrm{cm}^{-3} \mathrm{pc}$.

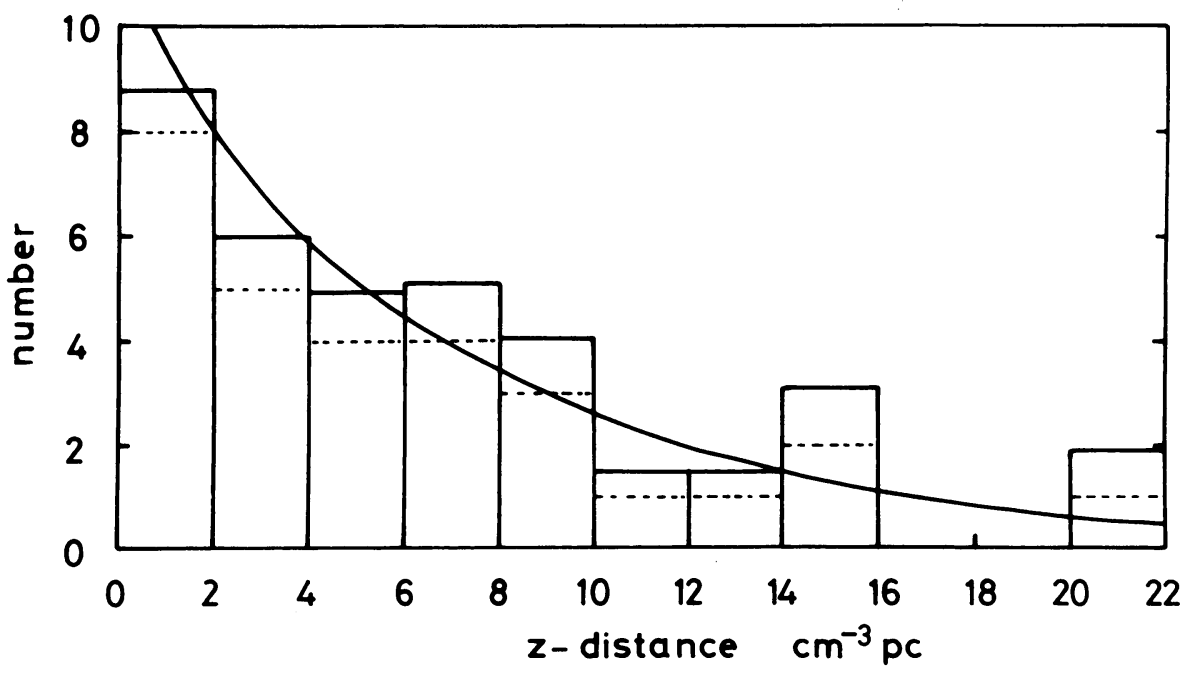

Fig. 7. The distribution, $Q_{3}(z)$, of pulsars in galactic $z$-distance derived from the observed distribution of Molonglo pulsars (shown dotted) and calibration data. Distance is expressed in terms of dispersion measure, in units of $\mathrm{cm}^{-3} \mathrm{pc}$. The distribution is adequately represented by an exponential of scale height $7 \mathrm{~cm}^{-3} \mathrm{pc}$. 
shifted slightly towards longer periods corresponding to the more numerous low luminosity pulsars.

\section{B. THE DISTRIBUTION IN LUMINOSITY}

It is apparent from Figure 6 that the numbers of pulsars per unit volume of space in each logarithmic interval of luminosity falls rapidly with luminosity. A power law of the form

$$
\varrho(L) \mathrm{d} L \propto L^{-x} \mathrm{~d} L
$$

represents the data moderately well with $x=2$. However the errors derived from assuming a Poisson distribution in each class of the observed distribution show quite clearly that the luminosity function is curved, with $x$ increasing from about 1.5 at low luminosity $\left(L=1 \mathrm{fu}(\mathrm{dm})^{2}\right)$ to about 3 at high luminosity $\left(L=10^{4} \mathrm{fu}(\mathrm{dm})^{2}\right)$.

The form of this luminosity curve is very little affected by the assumption that period and luminosity are unrelated. If the degree of correlation indicated in Figure 4 is allowed for, the change in the derived luminosity curve is within the random error limits, with marginally fewer low luminosity pulsars, and more high luminosity ones.

The overall scaling and general form of the luminosity curve have been experimentally confirmed in a recent pulsar search at Jodrell Bank (Davies et al., 1970). A search was made of 431 points within $1^{\circ}$ of the galactic plane at $408 \mathrm{MHz}$, analysing for periodicities in 13 minutes data from each point. The luminosities of the seven pulsars detected (of which 5 were new discoveries) are plotted as a (cross-hatched) histogram in Figure 8. The plain histogram represents the predicted numbers and luminosities expected using the Molonglo luminosity curve (Figure 6) and the distribution in $z$ (Figure 7). The dotted histogram shows the predicted numbers supposing all 431 points of observation had been exactly on the galactic plane. The excellent agreement between the predicted and observed distributions provides independent confirmation that the pulsar luminosity curve is reasonably well established at $408 \mathrm{MHz}$.

\section{THE DISTRIBUTION IN $z$-DISTANCE}

Comparison of the derived distribution in $z$ with the observed distribution shows that they are almost identical. The distribution is adequately described by an exponential function of scale height $7 \mathrm{~cm}^{-3} \mathrm{pc}$ in dispersion measure units, and this result is very little affected by selection effects. It cannot therefore be appreciably in error as a result of assuming the distributions in $P, L$ and $z$ to be independent. If $\bar{n}_{e}$ is taken to be $0.05 \mathrm{~cm}^{-3}$, the scale height is $140 \mathrm{pc}$.

\section{Interpretation}

\section{A. THE NUMBER OF PULSARS IN THE GALAXY}

The pulsar luminosity function shown in Figure 6 gives the number of pulsars per unit volume in each logarithmic interval of apparent luminosity. It is probable that pulsars have a finite beamwidth in latitude (i.e. in the plane containing the rotation 
axis) so that only a fraction are seen with their peak luminosity. The remainder will be seen in weak side lobe radiation or not at all. In order to make some estimate of how such 'latitude beaming' might affect the observed luminosity function, I have considered two models of the beam in latitude. One model, defined by Equations 6 ,

$$
\begin{aligned}
L & =L(0)\left(1+\left(\theta / \theta_{0}\right)^{2}\right)^{-1} \\
\theta_{0} & =5^{\circ}
\end{aligned}
$$

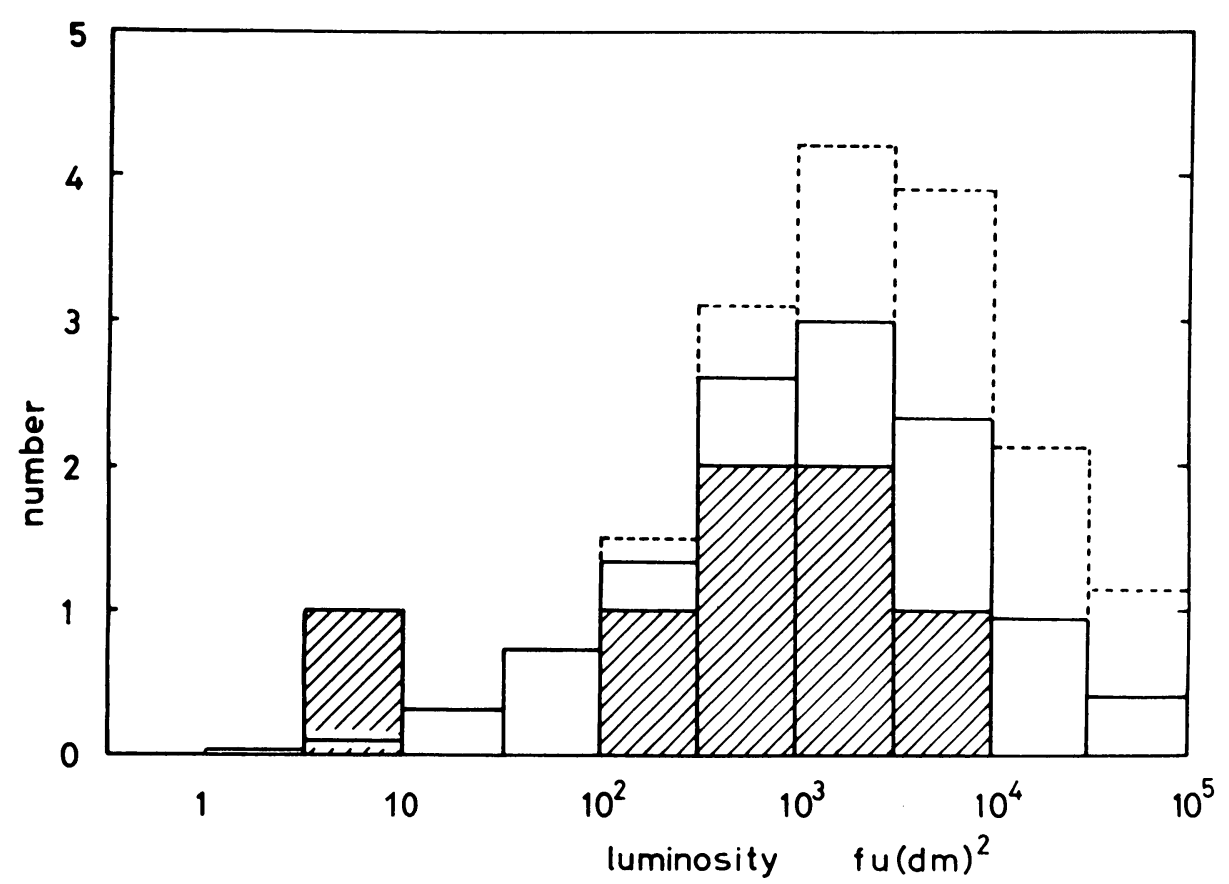

Fig. 8. The cross-hatched histogram shows the luminosity distribution of 7 pulsars observed in a recent $408 \mathrm{MHz}$ search at Jodrell Bank. The plain histogram shows numbers predicted for this search using the distributions derived from the Molonglo searches. The agreement confirms the general features and scaling of the derived distributions. The dotted histogram shows the predicted numbers that would have been observed if all the Jodrell observations had been confined to $b=0^{\circ}$.

is intended to represent a radiation pattern that remains reasonably strong in the side lobes. The second model defined by the gaussian function

$$
\begin{aligned}
L & =L(0) \exp -\frac{1}{2}\left(\theta / \theta_{0}\right)^{2} \\
\theta_{0} & =5^{\circ}
\end{aligned}
$$

represents a beam with very little side lobe radiation.

For both models I have computed a luminosity function in terms of $L(0)$ that would produce the distribution of apparent luminosities shown in Figure 6, supposing pulsar axes to be randomly orientated. The results are plotted in Figure 9 which shows the apparent luminosity curve as well as those derived in terms of $L(0)$ for 
the two models. The number scale is now expressed in terms of number per cubic $\mathrm{kpc}$ in each interval of 0.5 in $\log _{10} L$ based somewhat arbitrarily on taking the mean electron density in the galaxy to be $\bar{n}_{e}=0.05 \mathrm{~cm}^{-3}$. In terms of $L(0)$ there are several times more pulsars in each luminosity interval, and the curvature of the luminosity curve is increased.

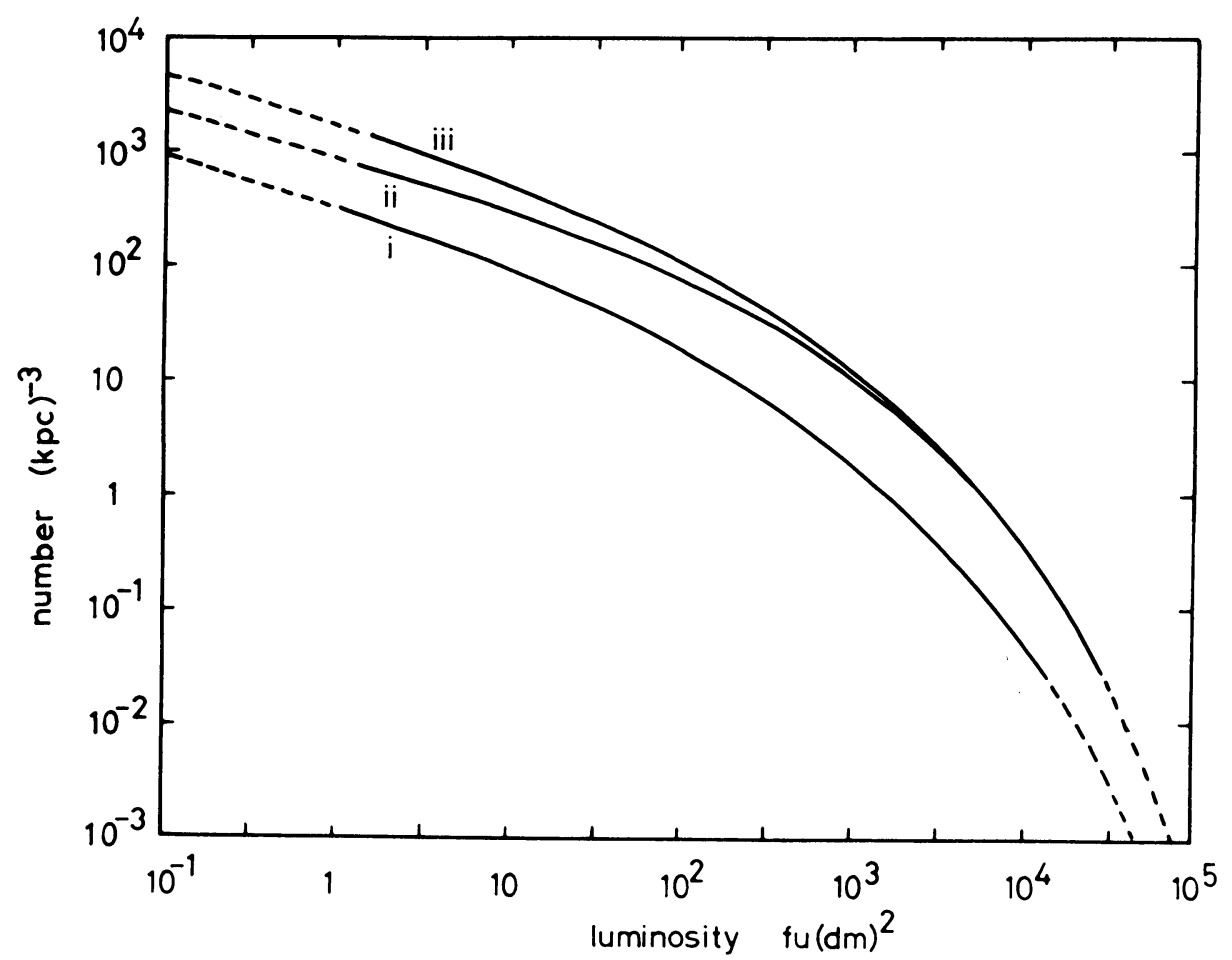

Fig. 9. Luminosity functions showing the number of pulsars in each interval of 0.5 in $\log _{10} L$ per cubic kiloparsec of the galaxy. The scaling is based on assuming that 1 dispersion measure unit is 20 parsecs, i.e. $\bar{n}_{e}=0.05 \mathrm{~cm}^{-3}$. Curve (i) is the distribution of apparent luminosities. Curve (ii) represents the distribution of peak luminosities assuming a beam in latitude given by $L=L(0)$ $\left(1+\left(\theta / \theta_{0}\right)^{2}\right)^{-1}$ with $\theta_{0}=5^{\circ}$. Curve (iii) is similar to (ii) but calculated for a gaussian beam $L=L(0)$ $\exp -\frac{1}{2}\left(\theta / \theta_{0}\right)^{2}$, again with $\theta_{0}=5^{\circ}$.

It is now possible to estimate the total number of pulsars in the Galaxy with luminosity greater than a suitably chosen lower limit. Using a value of mean electron density $\bar{n}_{e}=0.05 \mathrm{~cm}^{-3}$ and the gaussian model of the beam in latitude (Equation 7) the luminosity curve Figure 9 (iii) indicates that there are 2500 pulsars of luminosity greater than $L(0)=1 \mathrm{fu}(\mathrm{dm})^{2}$ per cubic kpc. Taking the scale height to be $140 \mathrm{pc}$ $\left(7 \mathrm{~cm}^{-3} \mathrm{pc}\right)$ in galactic $z$-distance and a value of $15 \mathrm{kpc}$ for the radius of the Galaxy, we obtain the result that there are $5 \times 10^{5}$ pulsars of peak luminosity exceeding $1 \mathrm{fu}(\mathrm{dm})^{2}$ in the Galaxy. This estimated number depends inversely on the assumed width of the beam in latitude, and directly on the square of the assumed mean electron 
density (not on $\bar{n}_{e}^{3}$ since the scale height in $z$-distance depends inversely on $\bar{n}_{e}$ ), and must as a result be regarded a good to within a factor of 10 only.

\section{B. COMPARISON WITH RATE OF OCCURRENCE OF SUPERNOVAE}

If it is assumed that there is a one-to-one correspondence between pulsars and supernovae, then the typical life-time of pulsars can be estimated from knowledge of the rate of occurrence of supernovae in the Galaxy and the total number of pulsars in the Galaxy. Taking the rate of occurrence of supernovae to be one every 50 years and the total number of pulsars to be $5 \times 10^{5}$ indicates that pulsars typically radiate with a luminosity in excess of $1 \mathrm{fu}(\mathrm{dm})^{2}$ for $\sim 2.5 \times 10^{7} \mathrm{yr}$. Since this is the order of life-time suggested by the observed rates of change of period of pulsars, it seems worth pursuing this argument in more detail.

Suppose that all pulsars are formed with a luminosity exceeding some value $L_{\min }$ and then evolve with continuously decreasing luminosity. Then in a steady state the number of pulsars $\mathrm{d} N$ in any luminosity interval $\mathrm{d} L$ (below $L_{\min }$ ) is given by the product of the rate of formation of pulsars $n_{0}$ and the time taken to evolve through the luminosity interval. Thus

$$
\mathrm{d} N=n_{0} \frac{\mathrm{d} t}{\mathrm{~d} L} \mathrm{~d} L=n_{0} \mathrm{~d} t .
$$

Integration gives

$$
\left(t_{2}-t_{1}\right)=\frac{1}{n_{0}}\left(N_{2}-N_{1}\right)
$$

where $\left(t_{2}-t_{1}\right)$ is the time taken for a pulsar to evolve from luminosity $L_{2}$ to $L_{1}$ and $\left(N_{2}-N_{1}\right)$ is the number of pulsars in the Galaxy between luminosities $L_{2}$ and $L_{1}$.

Figure 10 is a graph of the luminosity $\left(\log _{10} L\right)$ against $\left(1 / n_{0}\right) N(>L)$ where $N(>L)$ is the number of pulsars of luminosity greater than $L$. In view of Equation 9 , the graph can be regarded as representing the evolution of the luminosity of a typical pulsar below some (undetermined) luminosity $L_{\min }$. The abscissa of Figure 10 is a linear time scale in arbitrary units, $t_{0}$. The unit is $10^{7}$ years for the particular set of parameters we have been using, namely $\bar{n}_{e}=0.05 \mathrm{~cm}^{-3}, \theta_{0}=5^{\circ}$ and $n_{0}=\frac{1}{50} \mathrm{yr}^{-1}$ and is given more generally by

$$
t_{0}=\text { time unit }=10^{7}\left(\frac{\bar{n}_{e}}{0.05}\right)^{2}\left(\frac{5}{\theta_{0}}\right) \frac{1}{n_{0}} \text { years }
$$

It is not possible to determine the value of $L_{\min }$ from the present data, and the interpretation of the abscissa of Figure 10 as a time scale may be meaningless. However, inspection of the graph and also the luminosity curves (Figure 9) suggests to me that above luminosities of order $10^{1}$ to $10^{2} \mathrm{fu}(\mathrm{dm})^{2}$ we are seeing the creation and/or rapid evolution of pulsars, and below luminosities of $10 \mathrm{fu}(\mathrm{dm})^{2}$, the steady evolution of luminosity with a time scale of a few units, i.e. $\sim 10^{7} \mathrm{yr}$. 


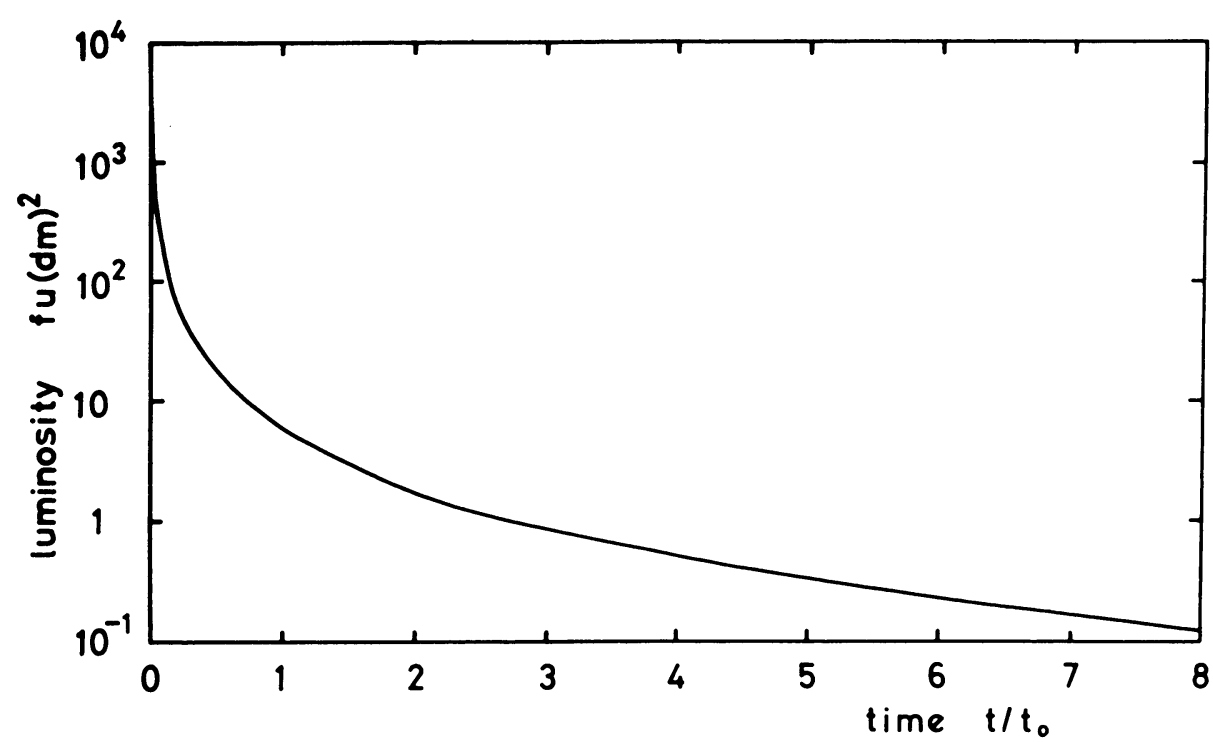

Fig. 10. An interpretation of the pulsar luminosity function (Figure 9 (iii)) in terms of the evolution in luminosity of individual pulsars. Assuming all pulsars are initially formed with luminosity in excess of $L_{\mathrm{min}}$, the curve represents the decay in luminosity below that luminosity. If pulsars are created with supernovae at the rate of about 1 every 50 years, the unit, $t_{0}$, of the time scale is $\sim 10^{7} \mathrm{yr}$. It is tentatively suggested that pulsars are generally created with luminosities in excess of $10 \mathrm{fu}(\mathrm{dm})^{2}$ and that below this luminosity, the curve indicates the time scale of their evolution in luminosity.

\section{COMPARISON OF THE $z$-DISTRIBUTIONS OF PULSARS AND SUPERNOVAE}

The supernovae appear to be concentrated in a thin layer in the galaxy. According to Poveda and Woltjer (1968) the mean $z$-distance $|\bar{z}|$ for nearby supernovae is $64 \mathrm{pc}$. Milne (1970) finds that more than half the supernovae in his compilation lie within $50 \mathrm{pc}$ of the galactic plane. The mean height of the pulsars above the galactic plane (see Figure 7) is $7 \mathrm{~cm}^{-3} \mathrm{pc}$ in dispersion measure units. Accordingly, if we are to match the two distributions it is necessary to assume that 1 dispersion measure unit corresponds with about $8 \mathrm{pc}$, implying a mean electron density of $0.12 \mathrm{~cm}^{-3}$. This value is slightly high compared with that deduced from the known dispersion and distance of the Crab pulsar $\left(0.03 \mathrm{~cm}^{-3}\right)$ but is consistent with the data for the Vela pulsar. In a recent note, Gold and Newman (1970) also note that a value of $\bar{n}_{e} \sim 0.1 \mathrm{~cm}^{-3}$ is required to match the thickness of the pulsar distribution to other galactic populations. Alternatively, the mean height of the pulsar distribution in $z$-distance may be significantly greater than that of the supernova remnants, either because they were formed at high velocities as Gunn and Ostriker (1970) and others have suggested, or because they are a different class of object.

\section{Conclusions}

The distributions of pulsars at $408 \mathrm{MHz}$ in period, luminosity and galactic $z$-distance 
are reasonably well represented by Figures 5,6 and 7. The principal difficulties in comparing these distributions with supernova data lie in the conversion from a dispersion measure distance scale to a geometric distance scale (involving knowledge of the mean electron density in the Galaxy) and in assessing the extent to which the pulsar radiation is concentrated in the plane containing the rotation axis. However by making reasonable estimates of these factors it is shown that the total number of pulsars in the Galaxy is consistent with the hypothesis that pulsars are formed at the same rate as supernovae, and that the pulsar luminosity decays with a time scale of order of $10^{7} \mathrm{yr}$. Uncertainties in the available data on the distribution of electrons in the Galaxy also limit the precision with which the height of pulsars above the galactic plane can be measured. As far as one can tell, the pulsars and supernovae extend over a similar range of $z$-distance, but it is possible that the pulsar distribution is slightly wider.

\section{Acknowledgements}

I thank Professor Sir Bernard Lovell and the University of Manchester for a Research Fellowship which enabled me to spend my year's sabbatical leave from the University of Sydney at Jodrell Bank.

\section{References}

Aizu, K. and Taketani, M.: 1970, Preprint, Rikkyo University, Tokyo.

Arnett, W. D.: 1969, Nature 222, 359.

Colgate, S. A. and White, R. H.: 1966, Astrophys. J. 143, 626.

Davies, J. G., Large, M. I., and Pickwick, A. C.: 1970, Nature 227, 1123.

Gold, T. and Newman, H. M.: 1970, Nature 227, 151.

Gunn, J. E. and Ostriker, J. P.: 1970, Astrophys. J. 160, 979.

Large, M. I.: 1970a, Astrophys. Letters 5, 11.

Large, M. I.: 1970b, Proceedings of Rome Conference on Pulsars and High Energy Activity in Supernovae.

Large, M. I. and Vaughan, A. E.: 1971, Monthly Notices Roy. Astron. Soc. 151, 277.

Maran, S. P. and Modali, S. B.: 1970, Earth Extraterest. Sci. (in press).

Milne, D. K.: 1970, Australian J. Phys. 23, 425.

Notni, P., Oleak, H., and Schmidt, K.-H.: 1970, Astrophys. Letters 6, 61.

Ostriker, J. P. and Gunn, J. E.: 1969, Nature 223, 813.

Poveda, A. and Woltjer, L.: 1968, Astron. J. 73, 65.

Prentice, A. J. R.: 1970, Nature 225, 438.

Prentice, A. J. R. and ter Haar, D.: 1969, Monthly Notices Roy. Astron. Soc. 146, 423.

Rickett, B. J.: 1969, Nature 221, 158.

\section{Discussion}

J. P. Ostriker: While I agree with many of your conclusions, I have some quibbles with your methodology. Why do you assume

$$
N(L, P, z)=A(L, P, z) \varrho(L, P, z) ?
$$

Must not the relation be

$$
N(L, P, z)=\iiint A\left(L^{\prime}, P^{\prime}, z^{\prime} ; L, P, z\right)\left(L,^{\prime} P^{\prime}, z^{\prime}\right) \mathrm{dL} L^{\prime} \mathrm{d} P^{\prime} \mathrm{dz} z^{\prime} ?
$$

M. I. Large: The degree of dependence of $P$ on $L$ is weak in the data available, and does not affect the analysis much. 
D. H. Staelin: A critical assumption is the relationship between distance and dispersion measure. What is the effect of HII regions upon the inferred distribution luminosities?

M. I. Large: I think the dispersion measure is a reasonable statistical indicator of distance and is justified by the large range in luminosity in my sample over a factor of $10^{4}$ which corresponds to $10^{2}$ in distance. Prentice and ter Haar showed that an $\mathrm{H}$ II region may increase the dispersion measure of a pulsar by a factor of 2 at the most.

J. A. Roberts: I would like to know whether there have been properly designed searches for radio pulsars in supernova remnants, which allowed for the expected large dispersion and short period by some form of dedispersing. Also, have there been searches for optical pulsars in the remnants, since optical searches are not subject to the problems of large dispersion coupled with short periods?

M. I. Large: In my opinion searches for pulsars in known supernovae have not been made with adequate sensitivity for periods $<0.25 \mathrm{sec}$.

D. Horowitz: In answer to Robert's question suggesting an optical search for pulsars near supernova remnants, I would like to say that our group at Harvard has been doing this for the past year and a half, and I will report on this later in the session.

J. E. Baldwin: What do you believe to be the relative $z$ distributions of the pulsars and the ionised material causing dispersion?

M. I. Large: I have assumed the pulsar $z$ distribution is narrower than the ionized layer. If the pulsar distribution is wider than that of the ionized layer then the real width of the pulsar distribution is greater than I have given in my paper. Already the pulsar distribution is wider than the supernovae.

J. V. Jelley: You mentioned there were $\sim 5.10^{5}$ pulsars in our own Galaxy. I would like to ask, if there were a comparable number in M31, would they contribute significantly to the total radio yield of this or other nearby galaxies?

M. I. Large: If there are a similar number of pulsars in M31, they would contribute negligibly to the continuous radio emissivity. Furthermore my luminosity curve is very steep at the high luminosity end. Extrapolation indicates that the most luminous pulsar in M31 would be quite undetectable with present techniques.

F. D. Drake: There have been discussions, particularly by Gold and Newman, of the apparent greater concentration towards the galactic plane of the shorter period pulsars. This seems to imply greater luminosity for the short period pulsars. If there is no dependence of luminosity on period, perhaps the long period pulsars have a greater mean $z$. What is your opinion?

M. I. Large: The effect described by Gold and Newman is plotted in Figure 11. I feel that the

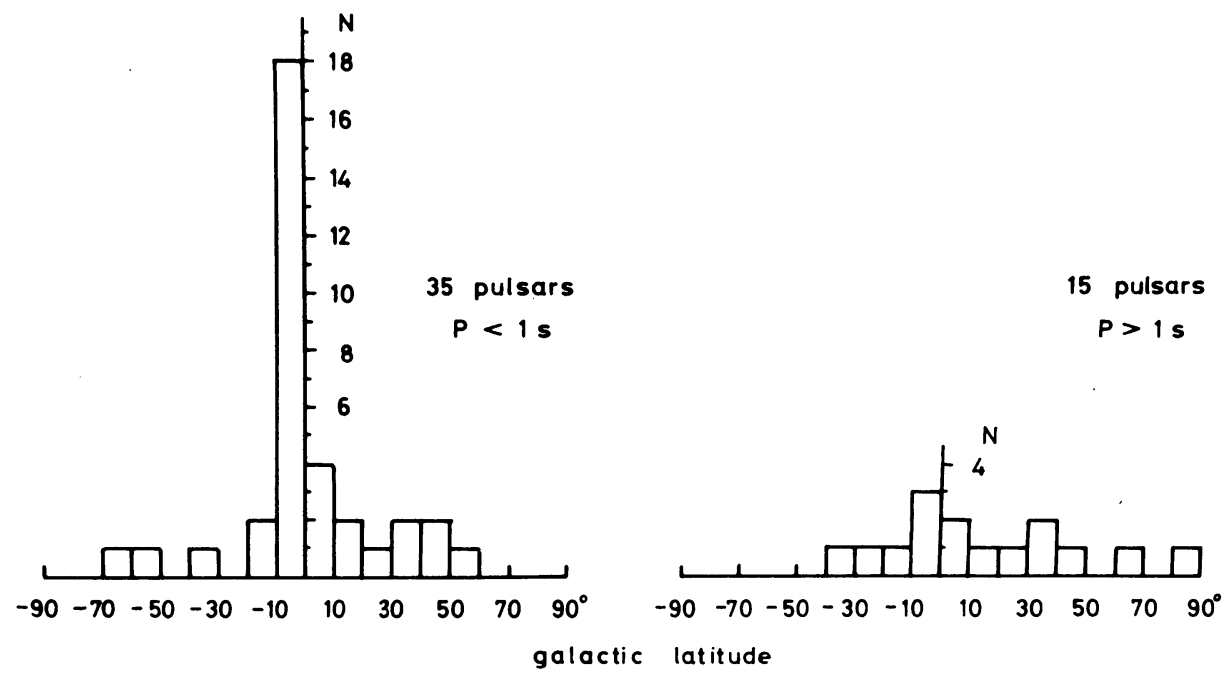

Fig. 11. The latitude distribution of known pulsars divided into (a) those with a period $<1 \mathrm{sec}$ and (b) those with a period $>1 \mathrm{sec}$. 
difference in the distribution of pulsars with $P<1 s$ and those with $P>1 s$ is not well established. An alternative presentation of the data is given in the top section of Figure 12 in which the Molonglo pulsars are shaded. The distribution for $P>1 s$ is multiplied by $\frac{35}{15}$ to make it comparable with the distribution for $P<1 s$ and it is seen that the distributions are not significantly different. If the range of periods is split at $P=0.7 \mathrm{sec}$ so that there are 25 in each class then there is no significant difference between the two distributions as shown in the bottom half of Figure 12.

Notni, Oleak and Schmidt conclude there is a correlation between period and galactic latitude at the $5 \%$ level. The data is given in Figure 13 where the Molonglo pulsars are shown as open circles and the Rest of the World as filled circles. Because of the observing technique (particularly the frequency) used the Molonglo pulsars tend to have a shorter period and lower latitude than the others. This
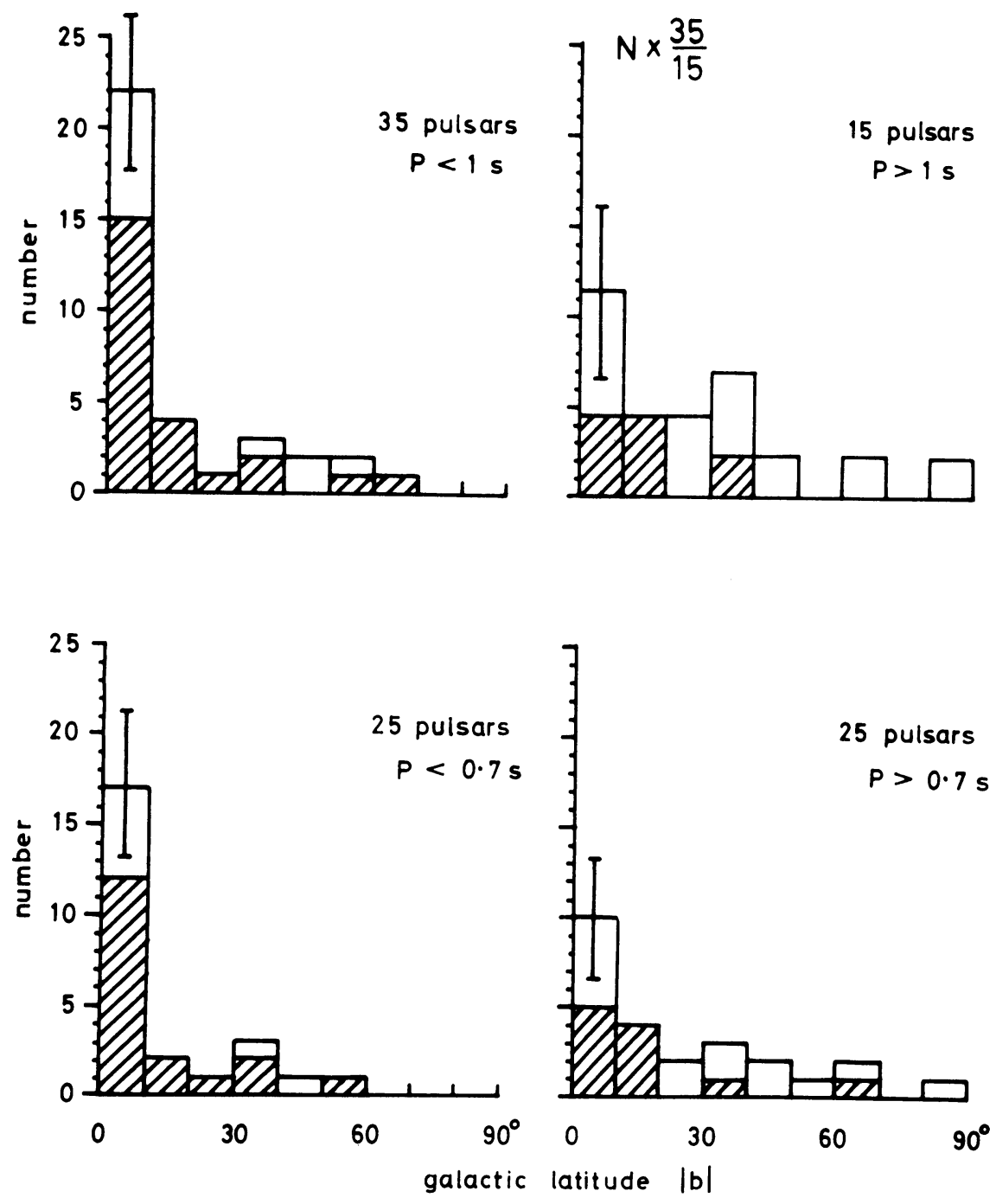

Fig. 12. The same data as in Figure 11, plotted as a function of $|b|$. The top half of the figure shows the normalized distributions. Error bars indicate the statistical uncertainty. The bottom half shows the data divided into equal groups with the division at a period of $0.7 \mathrm{sec}$. 
alone is responsible for some of the systematic trends in the statistics. A recent pulsar search at $408 \mathrm{HMz}$ using the Mk I telescope at Jodrell Bank discovered a number of low latitude, long period pulsars which when plotted on Figure 13 remove any correlation between period and latitude which may be present.

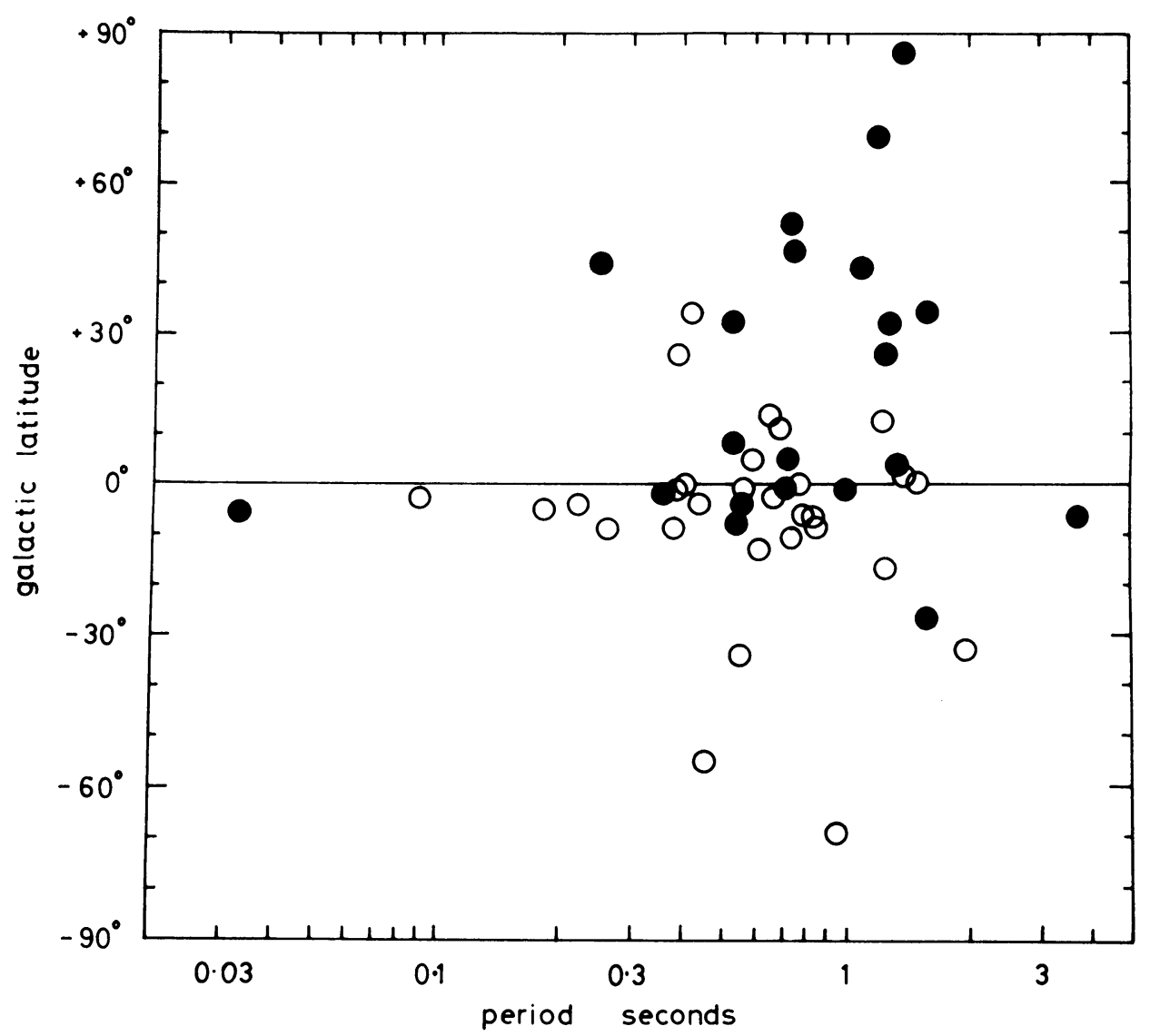

Fig. 13. A plot of the distribution of pulsars in period and galactic latitude. Molonglo pulsars are shown as open circles, the Rest of the World as filled circles. 